
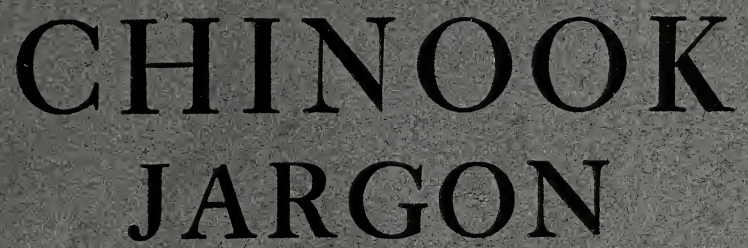

AS SPOKEN BY THE INDIANS OF THE PACIFIC COAST

For the Use of Missionaries, Traders, Tourists and Others Who Have Business Intercourse With the Indians.

\title{
CHINOOK-ENGLISH ENGLISH-CHINOOK
}

\section{PN} 846 . $\mathrm{T} 38$ 1914 BY REV. C. M. TATE ANTH 


\section{British Columbia Permanent Loan Company}

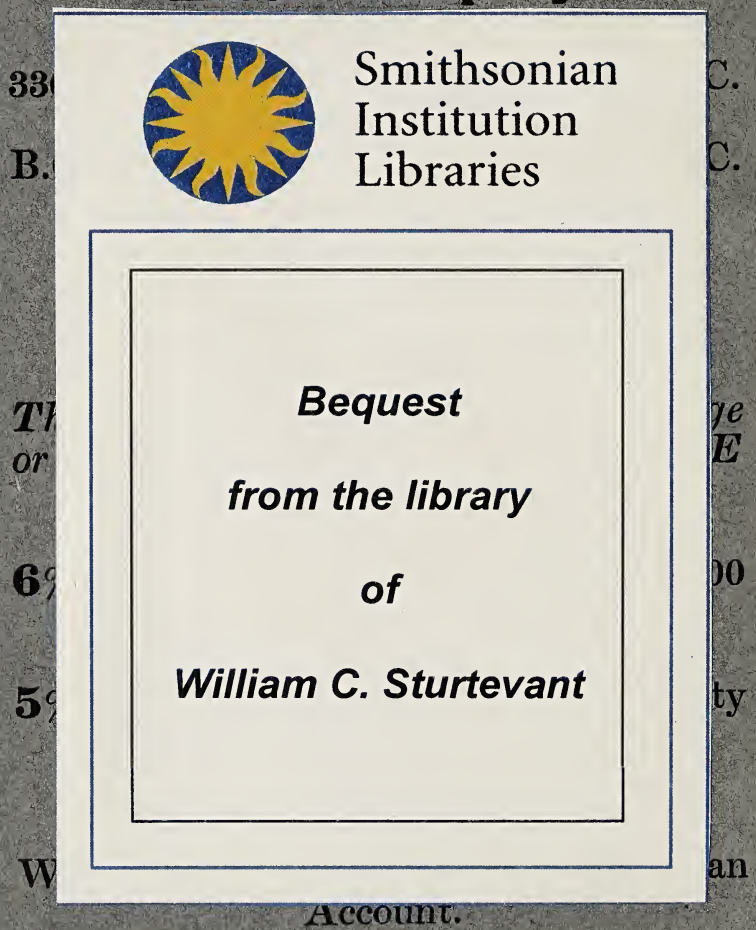

Prompt Attention Given Correspondence

$$
\begin{aligned}
& \text { T. D. MACDONALD, } \\
& \text { General Manager. }
\end{aligned}
$$




\section{CHINOOK JARGON}

\section{AS SPOKEN BY THE INDIANS OF THE PACIFIC COAST}

For the Use of Missionaries, Traders, Tourists and Others Who Have Business Intercourse With the Indians.

\section{Chinook-English English-Chinook}

BY REV. C. M. TATE

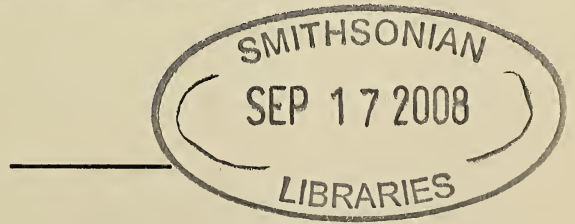

IOI4

Printed by Thos. R. Cusack, Victoria, B. C. 


\section{P R E F A C E}

In issuing this revised Dictionary of the Chinook Jargon, the object is to place in the hands of those who have business intercourse with the Indians of the Pacific Coast, as well as to tourists and others, a means of making themselves understood by the natives where the Chinook is spoken. This work has been carefully revised; the spelling simplified, and the arrangement of words and sentences concentrated, so that the pronunciation, and the way in which the words are used may at once be seen.

Victoria, B.C., March 1st, 1914.

Entered according to Act of Parliament of Canada, in the Year 1889, by M. W. Waitt, in the Office of the Minister of Agriculture. 


\section{REVISED DICTIONARY \\ OF THE}

\section{CHINOOK JARGON}

\section{Part I.- Chinook - English}

A.

Ah-ha, yes. Ah-ha nah-witka, yes, indeed.

Ahn-kut-te, formerly. Tenas ahnkutte, some time ago. Hyas ahnkutte, a long time ago. Delate ahnkutte, a very long time ago.

Al-ah, surprise.

Al-kie, presently; hold on. Tenas alkie, in a little while. Alkie nika klatawa, I will go presently.

Alta, now; at present. Nika skookum alta, I am strong now. Alta nika kumtux, now I understand.

A-mo-te, strawberry.

An-ah, expressing displeasure.

An-an-ah, expressing pain.

Ats, sister.

A-yah-whul, to borrow.

\section{B.}

Be-be, a kiss; to kiss.

Bed, a bed.

Bit or mit, a dime or $12 \mathrm{I} / 2$ cents.

Bloom, a broom.

Boat, a boat.

Boston, American. Boston man, American citizen.

Boston illahie, United States. Boston wahwah,

American talk, or English language.

Burdash, an hermaphrodite. 


\section{C.}

Calipeen, a rifle.

Can-aim, a canoe. Cooley canaim, canoe race. Haul canaim, pull up the canoe. Kokshut canaim, the canoe is split. Canaim stick, cedar wood.

Ca-poo, coat. Nika capoo, my coat.

Chah-ko, come; to become; came. Kah mika chahko,

where do you come from? Hyac chako, come quick. Chahko konamoxt nika, come with me.

Chack-chack, bald eagle.

Chee or Chick-ee, new; lately; just now. Chee chahko, just come. Hyas chee, very new. Delate chee, entirely new.

Cheet-woot, black bear.

Chick-a-min, money; iron and other metals. T'kope chickamin, silver. Pil chickamin, gold. Chickamin lope, wire or chain. Hyiu chickamin, plenty of money. Halo chickamin, no money.

Chick-chick, any wheeled vehicle.

Chil-chil, buttons.

Chitish, grandfather.

Chope, grandmother.

Cho-tub, flea.

Chuck, water; river; lake. Salt chuck, sea water. Skookum chuck, rapid water. Solleks chuck, angry water; rough sea. Mesatchie chuck, bad water. Snass chuck, rain water. Chuck chahko, tide coming up. Kilapie chuck, tide going back.

Chuck-kin, kick.

Clata-wa, go; go away.

Cly, cry.

Cole, cold. Hyas cole, very cold. Cole illahe, winter. Ickt cole, one year.

Comb, comb. 
Cooley, run or walk. Cooley cuitan, race horse. Skookum cooley, run hard. Cooley lapee, on foot. Yaka cooley copa cuitan, he went on horseback. Nika cooley copa chikamin wayhut, I am going by railroad.

Coop-coop, shell money (not in use).

Cuit-an, horse. Skookum cuitan, strong horse. Cultus cuitan, worthless horse.

Cush-oo, pig; bacon or pork. Siwash cushoo, seal. Cultus, worthless; good for nothing; no matter. Cultus copa mika, none of your business. Cultus copa nika, I don't care; no matter to me. Cu1tus wah-wah, worthless talk. Cultus cooley, taking a walk. Delate cultus, no manner of use.

\section{D.}

Delate, true; straight; direct. Delate nika wahwah, I am speaking the truth. Delate sick tumtum, very sorry.

Di-aub (or le-job, as spoken by the Indians) devil. Dily or dly, dry.

Doctin, doctor. Siwash doctin, Indian doctor or conjuror.

Dolla, money. Paipa dolla, bank notes. Pill dolla, gold. T'kope dolla, silver.

E.

Eh-kah-nam, story.

Eh-ko-li or kwun-nis, whale.

Ee-na, beaver. Eena stick, willow.

Ek-keh, brother-in-law. Nika sister yaka man, my sister's husband.

E-la-han or help, help; assist. 
E-lip, before; beginning; first. Nika muckamuck elip nika klatawa, I will eat before I go. Alkie nika elip clatawa, I will go first.

E-li-te, slave.

E-salth, Indian corn.

\section{G.}

Get-up, rise up; arouse. Mamook get-up yaka, rouse him up.

Glease, fat; oil ; grease. Hyiu glease, very fat. Halo glease, no fat; lean.

\section{$\mathrm{H}$.}

Hah-lakl, open. Mamook halakl lapote, open the door.

Haht-haht, mallard duck.

Halo, none; nothing; not. Halo mitlite, not in.

Halo muckamuck, no food. Halo nika tikke, I don't want.

Haul, draw ; pull. Haul skookum, pull hard.

Hee-hee, laugh; play. Heehee house, theatre; circus; any place of amusement.

Hoh-hoh, cough.

Hool-hool, mouse. Hyas hoolhool, rat.

House, house. Tipso house, barn. Mah-kook house, store. Kee-kwilly house, underground house.

Howh, hurry.

How-kwutl, unable.

Hul-lel, shake.

Hul-o-i-ma; different, strange; another. Huloima

iktah, strange thing. Huloima lalong, different language. Huloima man, another man.

Humm, smell or stink. Hyas humm, great smell.

Kloshe humm, good smell.

Hunl-kik, crooked or knotty. 
Huy-huy, change or exchange. Huyhuy mika capoo, change your coat. Kloshe nesika huyhuy musket, let us exchange guns.

Hwah, surprise.

Hyak, be quick; hurry. Hyak cooly, run or walk quickly. Hyak chahko, come quickly.

Hyas, big; great; very. Hyas kloshe, very good. Hyas kee-kwillie, very deep. Delate hyas, extremely large. Nika hyas tik-ke, I want it very much. Hyas makook, very dear; great price.

Hiyu, plenty ; much ; many ; enough. Hiyu chikamin, plenty of money. Hiyu canaim, many canoes. Hiyu snass, much rain. Wake hiyu, not many. Hy-kwa, shell money (large pieces).

\section{I.}

Ik-kik, fish hook.

Ikpooie, shut.

Ikt, one; once. Ikt moon, one month. Ikt Sunday, one week. Ikt sun, one day. Ikt dolla, one dollar.

Ik-tah, what. Iktah mamook? what's the matter? Iktah mika mamook? what are you doing? Iktah mika tikke? what do you want?

Ik-tahs, goods. Nika hiyu iktahs, I have plenty of goods. Yaka halo iktahs, he has no goods.

Il-la-hie, land; dirt; the earth. Sagh-a-lie illahie,

Heaven. Mesatchie illahie, bad soil.

In-a-poo, louse.

In-a-ti, beyond; across; on the other side. Tenas inati, a little beyond. Inati wayhut, across the road. Yaka mitlite inati. He is on the other side. Lolo nika inati chuck, take me across the river. 
Ipsoot, secrete; conceal; hide; cover up. Kah yaka ipsoot, where is he hiding. Ipsoot mamook, secret work.

Is-ick, paddle. Potlatch nika isick, give me a paddle. Halo isick, no paddles. Mamook isick, to paddle. Isick stick paddle wood; mapie; ash; cypress, yew.

Iskum, get; take. Potlatch nika; give me. Iskum, take it. Kah mika iskum? where did you get it?

Nika iskum copa stick, I got it in the woods.

It-lan, fathom.

It-lo-kum, gamble.

Itl-willie, flesh; meat. Moosmoos itlwillie, beef.

Nika itlwillie my flesh.

Its-wooat, black bear. Itswooat pesissie, dark blankets or cloth.

\section{K.}

Kah, where; whither; whence. Kah mika clatawa? where are you going? Kah yaka mitlite? where is he? Kon-a-way kah, everywhere.

Kah-kah, crow.

Kah-kwa, like; similar; the same. Delate kahkwa, exactly the same. Kloshe kahkwa that will do. Halo kahkwa, not like that. Kopet kahkwa, that is all.

Kah-na-way, acorns. Kahnaway stick, oak.

Kahp-ho, brother or sister.

Kah-ta, how. Kahta hiyu? how many? Kahta siah? how far? Kahta mika? how are you? Kahta mika mamook okook? how do you do that?

Kal-ak-a-lah-ma, goose.

Kal-a-kwah-tie, the inner bark of the cedar; petticoat

Kal-i-tan, an arrow, also applied to shot or bullets. 
Kamas, a sweet bulbous root.

Kamo-suk, beads. Tyee kamosuk, the large blue glass beads.

Kap-swalla, steal. Kapswalla klootchman, adultry. Kat-suk, middle or centre.

Kaw-py, coffee.

Ka-wak, to fly.

Kaw-ka-wak, yellow or light green.

Kee-kwil-lie, below; under; down; low ; deep. Kee-

kwillie chuck, low tide or deep water. Keekwillie copa chuck, under the water. Masin keekwillie, throw down (from above). Mitlite keekwillie, stay down, or stay below. Hyas keekwillie, very deep. Delate keekwillie, extremely deep. Keep-woot, a sharp instrument; a needle; a thorn. Keh-loke, swan.

Keh-see, apron.

Keh-wa, because.

Kes-chie, notwithstanding; although.

Ket-lin, pot; kettle or pan.

Kila-kila, bird.

Kila-pie, upset; return; overturn. Mamook kilapie spose kopet, bring back when finished. Nika hyak kilapie, I will return quickly. Yaka canaim kilapie, his canoe upset.

Kil-it-sut, glass or flinty substances.

Kim-tah, after; behind; last. Yaka siah kimtah, he is away behind. Nika cooley kimta, I will walk behind, after, or last.

King George, English. King George illahie, England. King George waw-wah, English language. Ki-noot1, tobacco.

Kish-kish, to drive.

Ki-wa, crooked.

Ki-yah, entrails. 
Klah, to escape, or come into view. Kloshe mika klah, good you escape. Yaka chahko klah, he has come into view.

Klagh-anie, outside. Mash klaghanie, to put outside. Clatawa klaghanie, go out.

Kla-how-ya, salutation, used at either meeting or parting. Klahowya six, good day friend, or good bye friend.

Kla-how-yum, poor; pity; mercy; in need of help.

Mamook klahowyum nika, pity me; help me.

Ne-sika delate klahowyum, we are extremely poor.

Klah-wa, slow; to move slowly.

Klak, from or off.

Klak-sta, who; whose. Klaksta mika? who are you?

Klaksta mika tik-ke? whom do you want? Klaksta okook house? whose house is that?

Klak-wun, wipe or lick.

Klale, black, or very dark colored. Klale man, negro. Hyas klale, very black.

Klap, find or catch. Mika klap yaka? did you catch him? Yaka klap copa wayhut, he found it on the road.

Kla-pite, thread; twine.

Klaska, they; theirs; then. Klaska chahko alta, they have come now. Klaska okook, that is theirs. Konaway klaska, all of them.

Kla-whap, hole.

Klem-a-hun, to stab or spear.

Klilt, bitter.

Klik-a-muks, bramble berries.

Klik-wal-lie, brass.

Kla-min-a-whit, untruth; lie. Yaka quansum klaminawhit, he always lies. Nika wake klaminwhit, I am not lying. 
Klim-min, soft.

Klis-kwis, mat. Siwash kliskwis, Indian mat.

Klogh-klogh, oysters.

Klo-nass, doubtful; uncertain; perhaps. Klonass kah, uncertain where. Klonass yaka mitlite, perhaps he is in.

Klone, three. Klone tin-tin, three o'clock.

Klook, crooked.

Klootch-man, woman; of the feminine gender. Tenas klootchman, girl or little woman. Klootchman komux, female dog; slut. Klootchman moos-moos, cow.

Kloshe, good. Hyas kloshe, very good. Kloshe kah-kwa, that will do; enough. Kloshe nanitch, take care; look out.

Klugh, to tear or plough.

Kluk-ulh, broad, or wide.

Klup, deep. Klup illahe, deep hole. Klıp chuck, deep water.

Ko, to arrive at; to reach. Chee klaska ko, they have just arrived.

Ko-ko, knock.

Kok-shut, break or broken; to beat or whip. Nika issick kokshut, my paddle is broken. Yaka kokshut nika, he beat or whipped me.

Kom-ux, dog.

Kon-a-way ; all ; every. Konaway kah, everywhere ;

Konaway klak-sta, everybody. Konaway ikta, everything.

Kon-a-moxt, both; together; with. Klaska konamoxt, they are together.

Koo-sah, the sky.

Ko-pa, at ; in ; of ; to ; towards ; about; by. Cultus kopa nika, nothing to me. Cooley kopa canaim, go by canoe. Mitlite kopa house, in the house. 
Ko-pet, stop; finished; only. Kopet wawah, stop talking. Kopet ikt, only one.

Kow, or gow, make fast; tie; bale or bundle. Ikt kow, one bale. Mamook kow, to tie.

Kuitan, horse.

Kul-lagh-an, fence or corral.

Kull, hard; difficult; tough.

Kum-tax, to know; understand. Nika kumtax, I know. Halo nika kumtax, I don't know.

Kun-sig? how many? Kunsig sun? How many days? Kunsig tin-tin? What time?

Kush-is, stockings.

Kwahn-sum, always.

Kwah-nice, whale.

Kwah-ta, twenty-five cents.

Kwah-tin, belly.

Kwaist, nine.

Kwalal, to gallop.

Kwal'h, aunt.

Kwan-kwan, glad.

Kwass, fear; afraid. Mamook kwass, to frighten.

Halo kwass, not afraid.

Kwates, sour.

Kweh-kweh, mallard duck.

Kwe-kwi-ens, a pin.

Kwe-kwe, finger ring.

Kwelth or youtl, proud.

Kwin-num, five. Mamook kwinnum, to count.

Kwish, refusal with contempt.

Kwit-shad-ie, rabbit.

Kwo-lahn, ear or to hear.

Kwult'h, to hit or wound.

Kwutl, to push or sneeze. 


\section{L.}

La-bleed, a bridle.

La-boosh, the mouth.

La-boo-ti, a bottle.

La-cal-at, a carrot.

La-ca-set, a box or trunk.

La-clo-ah or lu-kwa, a cross.

Lagh, to bend over; to stoop, to lean.

La-gum, pitch or gli1e. La-gum stick, pitch pine or any wood that is saturated with pitch.

La-gwin, a saw.

La-hal, to gamble with small round sticks, beaver teeth or pieces of bone.

Lahb, the arbutus.

La-hash, an axe. Tenas lahash, hatchet.

Lah-kit, four. Lah-kit tahtelum, forty.

La-kles, fat, oil.

L.a-lah, to cheat or deceive, to practice jokes.

La-lahm, an oar. Mamook lalahm, to row.

La-lim, a file.

La-long, tongue, language.

Lam, rum or other spirituous liquors.

La-messe, the ceremony of the mass.

La-ma-chin, medicine.

Lam-mi-eh, old woman.

La-mon-ti, mountain.

La-peep, tobacco pipe.

La-pesh, pole.

La-pel-lah, roast.

La-pelle, shovel or spade.

La-pe-use, mattock or hoe.

La-piege, trap.

La-plash, a board; lumber. Siwash laplash, boards split from the cedar.

La-po-el, frying pan. 
La-pool, chickens. Siwash la-pool, grouse.

La-poo-shet, fork.

La-poht, door.

La-san-jel, belt or girth.

La-sall, saddle.

La-shal-loo, plough.

I.a-shan-del, candle.

La-shase, chair.

La-shen, chain.

La-siet, plate.

La-sway, silk

La-tahb, table.

La-tet, head.

La-tlah, noise:

La-wen, oats.

La-west, vest.

Lay-lee, long time.

I.azy, lazy.

Le-bah-do, single.

Le-bal, ball or bullet.

Le-bisque, biscuit, cracker.

Le-blou, chestnut or sorrel colorerl.

Le-clem, cream colored.

Le-cock, male fowl; rooster.

Le-doo, finger.

Le-gley, gray colored.

Le-jaub, or diaub, the devil.

Le-kley, key.

Le-klo, nail.

L.e-koo, neck.

Le-kye, spotted.

Lo-lo-ba, ribbon.

Lee-loo, wolf.

Le-mah, hand or arm.

Le-ma-to, hammer. 
Le-mel, mule.

Le-mo-lo, wild.

Le-mo-to, sheep, mutton.

Le-pan, bread.

Le-pee, foot.

Le-pish-e-mo trappings of a saddle horse.

Le-plate, priest or minister.

Le-pwan, peas.

Le-sack, pouch, bag or sack.

Le-zep, egg ; eggs.

Lee-se-blo, spurs.

Le-see-zo, scissors.

Le-sook, sugar.

Le-tah, teeth.

Le-whet, whip.

Lice, rice.

Lik-pu-hu, elder sister.

Lip-lip, boil.

Lo-lo, carry; lead or take. Lolo yaka kopa mika house, take him to your house. Lolo kopa mika lemah, take, or carry it in your hand.

Lo-wul-lo, round or whole; unbroken.

Lope, rope. Siwash lope, rope made from cedar bark.

Luk-11t-chee, clams.

\section{M.}

Mah-kook, to buy or sell. Nika tikke mahkook, I want to buy. Kah mika mahkook, where did you buy. Mahkook house, store.

Mah-lie, to forget.

Mah-sie, thank you.

Maht-lin-nie, out at sea; off shore.

Maht-wil-lie, close to hand; in shore; keep in.

Malah, tinware, or crockery. 
Mal-lieh, to marry; married.

Ma-ma, mother.

Mam-ook, work; make; to do anything. Mamook issick, to paddle. Mamook haul, to pull. Mamook delate, make straight.

Man, man; of the male species. Tenas man, boy; small man. Man moos-moos, bull.

Mash, to throw away; to give up; to leave. Mash kopa illahe, throw it on the ground. Moxt sun nesika mash, we left two days ago. Mika tikke mash? do you want to give it up?

Mel-a-kwa, mosquitoes.

Mel-ass, molasses.

Mem-a-loose, die; dead. Wake siah memaloose, nearly dead. Mamook memaloose, to kill.

Mesah-chie, bad, wicked. Hyas mesahchie, very bad.

Me-si-ka (plural), you; your, yours.

Mika (singular), you or thou; thy; thine.

Mi-mie, down stream.

Mit-ass, leggings.

Mit-lite, sit down; at home; stay. Yaka mitlite, he is at home. Mitlite yah-wa, over there. Kloshe mitlike kopa nesika, stay with us.

Mit-whit, stand; stand up.

Moo-la, mill. Stick or la-plash moola, saw-mill.

Moolock, elk.

Moon, moon. Ikt moon, one month. Konaway moon, every month.

Moos-moos, horned cattle; buffalo.

Moosum, sleep.

Mow-itch, deer; venison.

Muck-a-muck, food; eat; bite; drink. Muck-a-muck chuck, drink water. 
Musket, gun. Stick musket, bow. Stone musket, flint lock gun.

\section{N.}

Nah, look here; to call attention. Nah! kah mika chahko? say! where are you from?

Nanitch, look; see; seek. Kloshe nanitch, look out; take care. Nika tikke nanitch, I want to see.

Nauits, beach, sea shore.

Na-wit-ka, yes, certainly, to be sure.

Nem, name.

Ne-nam-ooks, land otter.

Ne-si-ka ,we; us; our; ours.

Ne-wha, let me see; bring it hither.

Nika, I; me; mine.

Nose, the nose; point of land.

\section{O.}

Okook, this; that. Klaksta okook, who is that, or whose is that. Okook sun, today. Okook illahe, this place, or this world.

Ole-man, old man; old.

Ol-hy-iu, seal.

O-lil-lie, berries.

O-lo, hungry. Olo chuck, thirsty.

O-luk, snake.

Oos-kan, cup or bowl.

O-pe-kwau, basket.

O-pitl-kegh, bow.

O-pit-sah, knife.

O-poots, the posterior; the tail of an animal; stern of a boat.

Ow, brother. 


\section{P.}

Pahtl, full. Pahtl-lam, full of rum; drunk. Pahtl chuck, full of water.

Pay-pa, paper; book; letter; document.

Pent, paint.

Pa-pa, father.

Pa-si-ooks, Frenchmen; Catholic.

Pa-sis-sie, blanket.

Pe-chugh, green.

Pee, and ; then ; besides; but.

Pel-ton, fool; foolish; immoral; bad character.

Pe-shak, bad.

Pe-what-tie, thin.

Piah, fire; ripe, crooked. Piah olil-lie, ripe berries. Piah house, house on fire. Piah sick, venereal disease.

Pil, red.

Pil-pil, blood.

Pish, fish.

Pit-lilh, thick.

Piu-piu, skunk.

Poh, to blow out.

Po-lak-a-lie, night; dark. Tenas polakalie, evening. Sit-kum polakalie, midnight.

Po-lal-lie, gunpowder.

Poo, shoot; the sound of a gun. Mamook poo, to shoot.

Poo-lie, rotten.

Potlatch, a gift; give; giving away blankets. Potlatch nika, give me.

Powitch, crab apple.

Puk-puk, fist-fight. Hee-hee puk-puk, to box.

Puss-puss, cat. Siwash puss-puss, wild cat. Hyas puss-puss, panther or coughar. 
$\mathrm{S}$.

Sagh-a-lie, high; above; up. Saghalie tyee, God, or chief above. Saghallie illahie, heaven.

Sail, a sail; cotton or linen goods. Tzum sail, cotton print. Mamook sail, make sail. Mash sail, take down the sail.

Sa-kah-leks, pants. Keekwillie sakahleks, drawers. Sal-lal, the sal-lal berry.

Salmon, salmon.

Salt, salt. Salt chuck, sea water; the ocean.

San-de-lie, ash colored; roan colored.

Sap-o-lel, wheat; flour. Piah sapolel, cooked flour or bread.

Scotty, crazy. Scotty-house, lunatic asylum.

Se-ah-host, face, eyes.

Se-ah-po, hat or cap.

Shame, shame. Halo mika shame, are you not ashamed?

Shan-tie, to sing.

She-lokum, looking glass.

Ship, ship. Ship man, white man; sailor.

Shirt, shirt.

Shoes, shoes. Skin shoes, mocassins.

Shot, shot; lead.

Shu-gah, sugar.

Shugh, a rattle. Sugh opoots, rattlesnake.

Shwah-kuk, frog.

Siah, far. Wake siah, not far. Hyas siah, very far.

Delate siah, a great distance.

Siam, grizzly bear.

Sick, sick. Sick tum tum, grieved, sorry, unhappy. Sin-a-moxt seven.

Si-pah, straight.

Sis-ki-you, bob-tailed horse. 
Sit-kum, half. Sit-kum dolla, half dollar. Sit-kum sun, noon.

Sit-lay, stirrups.

Sit-shum, swim.

Si-wash, Indian.

Six, friend.

Skin, skin ; buck-skin; fur, as beaver-skin, etc. Skinstick, bark of a tree.

Skookum, strong. Skookum house, jail. Skookum chuck, rapid.

Skwis-kwis, squirrel.

Smoke, smoke; fog, clouds.

Snass, rain. Snass chuck, rain water. Snass wind, southeast wind.

Snow, Snow.

Soap, soap.

So-le-mie, cranberry.

Sol-leks, anger, angry.

So-pe-na, jump.

Spo-oh, to fade, faded.

Spoon, spoon.

Spose, suppose ; if ; provided that. Spose mika tikke, if you want. Spose yaka chahko, if he comes.

Stick, firewood; a tree ; wooden articles; yard measure. Ikt stick, one yard. Lolo pia stick, bring some firewood.

Stocken, stockings or socks.

Stoh, loose ; untie.

Stone, rock or stone; bone; testicles.

Stote-kin, eight.

Stut-chin, sturgeon.

Suk-wa-lash, gun.

Sun, the sun; day. Tenas sun, morning. Sit-kum sun, noon. Klup sun, sunset. Moxt sun, two days. 
Sunday, Sunday; one week. Moxt Sunday, two weeks.

\section{T.}

Tagh-um, six.

Tahl-kie, past. Tahlkie sun, yesterday. Tahlkie warm, last year or last summer. Tahlkie cole, last winter.

Tah-nim, to meaure.

Tah-tlum, ten. Klone tahtlum, thirty. Tahtlum pe ikt, eleven, etc.

Tal-a-pus, prairie wolf; sneak, when applied to a person.

To-mah-no-us, witchcraft; magic; supernatural; spirit.

Ta-mo-litch, barrel or tub.

Tanse, to dance.

Tea, tea.

Tea-whit, the leg.

Teh-teh, to trot.

Tenas, small; not much; litte; few ; a child. Tenas man, a boy. Hyas tenas, very small.

Te-pah, quills; the wing of a bird.

Tikke, want; wish; love; like. Ikta mika tikke? what do you want?

Tik-tik, a watch.

Til-i-kum, people. Nika tilikum, my people, or my relations. Huloima tilikum, strangers.

Till, tired; heavy. Mamook till, to weigh. Nika hyas till, I am very tired, or very heavy.

Tin-tin, a bell; a musical instrument; a clock. Kunsig tin-tin? what time is it? Mamook tintin, ring the bell.

Tip-so, grass or leaves. Tipso kila-kila, feathers. Tipso illahie, prairie. Dly tipso, hay. 
T'kope, white or very light colored. T'kope tilicum, white people.

Tl'kope, cut; hew, chop.

Toh, to spit.

Toke-tie, pretty.

To-lo, to win; to gain; to earn.

To-mol-la, tomorrow.

Tot, an uncle.

To-to, to shake, sift, or winnow.

To-toosh, milk; the breast of a female.

To-wagh, bright; shining; light.

Tsee-pie, mistake, blunder.

Tshi-ke, directly; soon.

Tsi-at-ka, the devil who roams at night.

Tsil-tsil, buttons. Saghalie tsil-tsil, the stars.

Tish, sharpen.

Tsole-pat, shot pouch.

T'so-lo, to wander in the dark; to feel lost.

Tsugh, a crack or split.

Tuk-a-mo-nuk, one hundred.

Tuk-wil-la, nuts.

Tum-tum, the heart; will ; mind ; opinion. Sick tumtum, sorry; grieved. Moxt tum-tum, undecided. Nika tum-tum kah-kwa, I think so. Kahta mika tum-tum, how do you feel or think.

Tum-wata, a waterfall.

Tup-shin, a needle.

Tyee, a chief.

Tzee, sweet.

Tzig-tzig, wagon, cart or other wheeled vehicle.

Tzum, to write or paint. Tzum sail, printed calico. 
W.

Wagh, to pour; to spill; to vomit.

Wake, no. Wake six, no sir, or no friend. Wake kah-kwa, not that way; not so.

Waki, tomorrow.

Wah-pit-to, potato.

Wash, wash. Mamook wash iktahs, wash the clothes. Wash mika lee-mah, wash your hands.

Waum, warm. Waum illahie, summer. Hyas waum, very warm. Delate waum, hot.

Wah-wah, to talk; speak; call; ask; tell; answer, etc.

Mesatchie wah-wah, bad talk. Hiyu wah-wah, too much talk. Cultus wah-wah, nonsense.

Way-hut, road or trail. Chickamin wayhut, railroad.

Whim, to fell or throw down.

Win-a-pie, by-and-by; presently.

Wind or win, wind; breath; breathe.

\section{Y.}

Yah-hul, a name.

Yaka, he; she; it; his; hers; him, etc.

Yah-kis-ilth, sharp.

Yah-wa, there, thence.

Yak-so, hair; hair of the head.

Yak-wa, here, this way.

Yak-wah-tin, entrails.

Yi-yem to relate; to preach; a story or tale.

Yout1, proud, envious.

Youtl-kut, long in length.

Yout-skut, short. 


\section{REVISED DICTIONARY OF THE CHINOOK JARGON}

\section{Part II. - English - Chinook}

A.

Above, sagh-a-lie.

Absolve, mamook stoh.

Acorns, kah-na-way.

Across, in-a-ti.

Admiration, hwah.

Afraid, kwass.

After, kimta.

Afterwards, kimta.

Again, waght.

All, kon-a-way.

Alms, e-la-han or e-lann.

Also, waght.

Although, kegh-tchie.

Always, kwansum.

American, Boston.

Among, kon-a-moxt.

Amusement, he-hee.

And, pee.
Anger, angry, sol-leks.

Another, hul-oi-ma.

Ants, kuckwalla.

Apple, le pom.

Apron, keh-su or i-suk.

Arbutus, lahb.

Arise, get up.

Arm, lee-mah.

Arrive at, ko.

Arrow, ka-li-tan.

Ash, isick stick.

Ashamed, shame.

Ask, wa-wah.

As if, kah-kwa, spose.

At, ko-pa.

Aunt, kwal'h.

Awl, shoes keep-wot.

Axe, le hash.

B.

Baby, pa-poose.

Back, kimta.

Bacon, cush-oo.

Bad, mesat-chie, or peshack.

Bag, le-sak.

Ball, le-bal.

Bargain, mak-kook; huyhoy.

Bark, skin stick.

Barley; la-reh.

Barrell, tah-molitch.

Basin, ketlin.

Basket, opekwan.

Beads, kamosuk.
Bear, (black) chet woot; its-woot.

Bear (grizzly) siam.

Beat to, kokshut.

Beaver, ee-na.

Because, kehwa.

Become to, chahko.

Bed, bed.

Before, e-lip or el-ip.

Behind, kimta.

Behold, nanitch.

Bell, tin-tin.

Belly, kwah-tin.

Below, keekwillie. 
Belt, la-sanjel.

Berries, olillie; olallie.

Best, elip kloshe.

Between, patsuk.

Beware, kloshe nanitch.

Beyond, yahwa.

Bible, saghalie tyee pay-pa.

Big, hyas.

Bird, kulakula.

Biscuit, lebiskwee.

Bit or dime, bit.

Bite, muck-a-muck.

Bitter, klihl.

Black, klale.

Blackberries, klikamuks.

Blanket, pasissie.

Blind, halo seahhost.

Blood, pilpil.

Blow out, mamook poh.

Blue (light) spooh.

Blue, (dark) klale.

Blunder to, tsee-pie.

Board, la-plash.

Boat, boat.

Bobtailed, siskiyou.

Body, itlwillie.

Boil to, liplip.

Bone, stone.

Borrow to, ayahwhul.

Both, konamoxt.
Bottle, labooti.

Bow, opitlkegh.

Bowl, ooskan.

Box, lacasett.

Bracelet, klikwallie.

Brass, klikwallie.

Brave, skookum tumtum.

Bread, piah sapolill.

Break to, kokshut.

Breasts, totoosh.

Bridle, la-bleed.

Bright, towagh.

Bring hither, mamook chahko.

Broad, klukluh.

Broom, bloom.

Broken, klook or kokshut.

Brother, kahpho, (if older than the speaker). Ow, (if younger).

Brother-in-law, ek-keh.

Bucket, tahmolitesh.

Buffalo, moosmoos.

Bullet, le-bal; kalitan.

Bundle, kow.

But, pee.

Butter, totoosh lakles.

Buttons, tsil tsil.

Buy to, mahkook.

By-and-by, winapie.

\section{C.}

Candle, la shandel.

Calf, tenas moosmoos.

Calico, tzum sale.

Canoe, canaim.

Carrot, la-calat.

Carry to, lolo.

Cart, tzig-tzig.

Cascade, tumwater.

Cask, ta-moo-litch.

Cat, puss-puss.
Cattle, moos-moos.

Cedar, canaim stick.

Certainly, nawitka.

Chain, la-shen; chickamin lope.

Chair, la-shase.

Change, huy-huy.

Christmas day, hyas Sunday

Christ, Jesu-kree.

Cheat, la-lah. 
Chicken, la-pool.

Chief, ty-ee.

Child, tenass.

Chimney, la-shumna.

Clams, lukutchee; ona.

Clock, tin-tin watch.

Cloth, sail.

Clouds, smoke.

Coal, pia stone.

Coat, cah-po.

Coffee, coh-py.

Cold, cole.

Color, tzum.

Comb, comb.

Come, chah-ko.

Conceal, ipsoot.

Confess, wau-wah; yiem.

Conjuring, ta-ma-na-wis.

Cook, cook.

Copper, pil chickamin.
Cord, tenas lope; whalim.

Corn, esalth.

Corral, kullaghan.

Cotton goods, sail.

Cough, hoh-hoh.

Count, mamook kwunnem.

Cow, klootchman moosmoos.

Coyote, talapus.

Crab apple, powitch.

Cranberry, solemie.

Crazy, scottie.

Cream colored, la clem.

Crooked, ki-wa.

Cross, la cloa.

Crow, kaw-kaw.

Cry, cly.

Cup, ooskan.

Curly, hunl-ki.

Cut, tl'kope.

\section{D.}

Dance, tanse.

Dark, pclakilie.

Day, sun.

Dead, memaloos.

Deaf, ikpooie qullan.

Dear (in price) hyas mah-kook.

Deep, klup.

Dear, mowitch.

Demon, skookum.

Devil, di-aub; lejob.

Different, huloima.

Difficult, kull.

Dig, kilapie illahie.

Dime, bit, or mit.

Dirty, pahtl illahie.
Dish, oscum.

Do, mamook.

Doctor, doctin.

Dog, koh-mux.

Dollar, dolla, or dah-1a.

Door, la-pote.

Doubtful, klonas.

Down stream, mie-mie.

Drink, muckamuck chuck.

Drive, kish-kish.

Drunk, pahtl-lam, or potlum.

Dry, dly.

Duck, kweh-kweh.

Dust, dly illahie, or kahkwa polalie.

E.

Eagle, chack-chack.

Ear, qullan.

Early, tenas sun.

Earn, tolo. 
Earth, illahie.

Eat, muck-a-muck.

Egg, la-sap.

Eight, stote-kin.

Elk, mooluck.

Enclosure, kullagan.

End, kimta, or opoots.

Enough, kloshe kah-kwa; hi-yu; kopet.

Fact, delate.

Fade, spo-oh.

Falsehood, klim-in-a-whit.

Far, siah.

Fast (quick), hyak.

Fast (tight), kwutl.

Fasten, kow.

Fat, glease; la-klees.

Father, papa.

Fathom; itlahn.

Fear, kwass.

Feathers, tipso kila-kila.

Feet, la-pee-ah.

Fell to, mamook whim.

Fence, kullaghan.

Fetch, mamook chahko.

Fever, waum sick.

Few, tenas; wake hi-yu.

Field, klackan.

Fight (with fists), puk-puk.

Fight (quarrel), mamook

\section{solleks.}

Figured, tzum.

File, la-leem.

Fill; mamook pahtl.

Find, klap.

Fine (good), kloshe.

Fingers, le-doo.

Finish, kopet.

Fire, piah.

First, elip.

Fish, pish.
English, King George.

Entrails, kyagh.

Evening, tenas polakilie.

Every, konaway.

Exchange, huy-huy.

Extinguish, mash; mamook poh.

Eyes, see-a-host.

F.

Fish-hook, ikkik.

Five, kwinnum.

Flag, Sunday sail.

Flea, sopen enapoo.

Flesh, itl-willie.

Flint, kil-it-sut.

Flour, sap-a-lel.

Fly to, ka-wak.

Fog, smoke.

Food, muckamuck.

Fool, scotty.

Foolish, scotty, or pelton.

Foot, le-pe-ah.

For, kopa.

Foremost, elip.

Forenoon, tenas sun.

Forever, kwahn-sum.

Forget, mahlie.

Forgive, stoh.

Fork, la-poo-shet.

Forty, la-kit tatlum.

Four, la-kit.

Fowl, la-pool.

French, pa-si-ooks.

Friend, six.

Frog, swah-kuk.

Fry, mamook lapoel.

Frying pan, lapoel.

Full, pahtl.

Fun, hee-hee.

Fur, skin. 
G.

Gain, tolo.

Gallop, kwal-lal.

Gather, hokumelk.

Get, iskum.

Get up, get up, or ketup.

Ghost, skookum.

Gift, cultus potlatch.

Girl, tenas klootchman.

Give; potlatch.

Glass, shelokum.

Go, klatawa.

God, saghalie tyee.

Gold, pill dolla.

Good, kloshe.

Good-bye, klahowya.
Goods, iktahs.

Goose, kilakilama.

Grandfather, chope.

Grandmother, chitish.

Grass, tipsoo.

Grease, glease; laklees.

Green, peechugh.

Grey, le-gley

Grieved, sick tum-tum.

Grizzly bear, siam.

Ground, illahee.

Grouse, siwash lepool.

Gun, musket.

Gunpowder, poo-lal-lie.
Hair, yakso

Half, sitkum.

Hammer, le-mah-to.

Hand, le-mah.

Handkerchief, hankatshum.

Hard, kull.

Hare or rabbit, kwitshadie.

Harrow to, mamook comb illahee.

Hat, seah-po.

Haul, haul.

Hawk, shak-shak.

Hay, dly tipso.

$\mathrm{He}$, yaka.

Head, la-tet.

Heart, tum-tum.

Heaven, saghalie illahee.

Heavy, till.

Help, mamook elann.

Hen, lapool.

Her, yaka.

Hers, yaka.
Here, yakwa.

Hide, skin.

High, saghalie.

His, yaka.

Hit, kwulh.

Hoe, la peosh.

Hog, cushoo.

Hole, kla-whop.

Hook, ik-kik.

Horse, ku-it-an.

House, house.

How, kah-ta.

How are you, kahta mika.

How large, kahta hyas.

How many, kahta hiyu.

How much, kon-sig.

Hundred, tuk-o-mo-nuk.

Hungry, o-lo.

Hurry, hy-ac howh.

Hurt, sick.

Husband, man.

Hush, kopet wah-wah. 
I.

I, or me, nika.

Ice, ice.

If, spose.

Ill, sick.

Immoral, pelton.

In, kopa.

Inability, how-kwutl.

Jail, skookum house.
Indian, Siwash.

Inshore, mahtwillie

Inside, mit-lite.

Iron, chickamin

Island, statejoy.

It, yaka.

\section{$\mathrm{J}$.}

Jealous, sick tum-tum.

$\mathrm{K}$.

Kamass root, la kamass.

Keg, tamoolitch.

Kettle, kettlin.

Key, la kley.

Kick, chuck-kin.

Kill to, mamook memaloos.

Kiss, bebe.

Knot (tree), le mah stick.

Knot (tie), kow

Knotty, hunl-ki.

Know, kumtux.

\section{L.}

Labor, mamook.

Lady, tyee klootchman.

Lamb, tenas la-moto.

Lame, klook teah-whit.

Lamp, lamp.

Land, illahee.

Language, la-long.

Large, hyas.

Last, kimta.

Laugh, hee-hee.

Lazy, lazy.

Lead, kalitan.

Leaf, tipso stick.

Leaf, sopena.

Lean (to), lagh.

Leave (go), klatawa, or mash.

Leave (off), kopet.

Leg, teah-whit.

Leggings, mitass.

Lend, len, or ayahwhul.

Lick, klahwhun.
Lie (to), kla-min-a-whit.

Lift, mamook saghalie.

Light, tooah.

Lightning, saghalie pia.

Like, kah-kwa.

Listen, ne-wha.

Little, tenas.

Long, youtl-kut.

Long (ago), lay-lee or ahn-kuttee.

Look, nanitch.

Looking glass, shlokum.

Look out, klosh nanitch.

Loose, stoh.

Louse, enapoo.

Love, hyas tikkee.

Low, keekwillie

Lower, mamook keekwillie.

Lumber, la-plash.

Lunatic, scotty.

Lunch, tenas muckamuck. 
M.

Magic, tamanawis.

Make, mamook.

Male, man.

Mamma, mamma.

Man, man.

Many, hi-yu.

Mark, tzum.

Marry, mallieya.

Mast, sail stick.

Master, tyee.

Mat, kliskwis.

Matches, matches.

Mattock, la-peosh.

Me, nika.

Meat, itl-willie.

Medicine, lamachin.

Men (many), hiyu man.

Mend, mamook tupshin.

Menstruate, mash pil-pil.

Middle, katsuk.

Midnight, sitkum polakilie.

Milk, to-toosh.

Mill, moola.

Mind, tum-tum.

Nails, lecloo.

Name, nem.

Narrate, yi-yem.

Near, wake siah.

Neck, le-cou.

Needle, keepwoot.

Negro, tlail man.

Never, wake kunsig.

New, chee or chikee.

Night, polakilie.

Nine, kwaist.

No, not, wake.

Oak, kull stick.

Oar, la-lum.
Mine, nika.

Miss, tzeepie.

Mistake, tzeepie.

Mocassins, skin shoes.

Molasses, melass.

Money, chickamin.

Month, moon.

Moon, moon.

More, waght.

Morning, tenas sun.

Most, elip hiyu.

Mosquito, malakwa.

Mother, mamma.

Mountain, lamonti.

Mouse, hool-hool.

Mouth, la-booshh.

Much, hiyu.

Mud, clenin illahee.

Mug, la-pot.

Mule, la-mel.

Musket, musket.

Mussels, to-luks.

My, myself, nika.

N.

Nobody, wake klaxta.

Noise, la-tlah.

None, halo.

Nonsense, cultus wah-wah.

Noon, sitkum sun.

Nose, nose.

Not, wake.

Nothing, halo ikta, kultus.

Notwithstanding, kaghtchie.

Now, alta.

Nuts, tuk-willa.

O.

Oats, la-wen.

Off, klak. 
Off shore, mahtlinnie.

Oil, glease.

Old, oleman.

Old woman, lammieh.

One. ikt.

Open, hah-lakl.

Opinion, tum-tum.

Opposite, inati.

Or, pe.

Other, huloima.

Paddle, issick.

Paint, paint.

Pants, se-gah-leks.

Panther, swa-wa.

Papa, papa.

Paper, pay-pa.

Part, sitkum.

Peas, le pwau.

People, tilicum.

Perhaps, klonas.

Petticoat, kwekwiens.

Pine, le gum stick.

Pipe, la peep.

Pistol, tenas musket.

Pitch, la gum.

Plank, la plash.

Plate, la siet.

Pleased, kloshe tum-tum.
Otter (land), nenamooks.

Our, nesika.

Outside, klaghanie.

Owl, waugh-waugh.

Over (above), saghalie.

Over (the other side), inati.

Overcoat, hyas capo.

Ox, moos-moos.

Oyster, klogh-klogh.

P.

Plough, le shalloo.

Plough to, klugh illahie.

Pole, pehsh.

Poor, klahowyam.

Pork, cusho.

Porpoise, tuiceco.

Posteriors, opoots.

Potato, wappatoo.

Pour to, wagh.

Pot, ketlin.

Powder, polallie.

Prairie wolf, talapus.

Presently, alkie; winapie.

Pretty, toketie.

Priest, la plete.

Proud, youtl; kwetl'h.

Provided that, spose.

Pull, haul.

Q.

Quarter, tenas sitkum.

Quarter (of a dollar), kwahta.

Quick, hyak.

Quills, tepeh.

\section{R.}

Rabbit, kwitchadie.

Race-horse, coolie kiuatan.

Rain, snass.

Raspberries, seahpult olillie.

Rat, hyas hoolhool.
Rattle, shugh.

Rattlesnake, shughopoots.

Razor-fish, ona.

Reach, ko.

Red, pil.

Relate to, yiem. 
Remain, mitlite.

Remove, mahsh.

Return to, kilapie.

Ribbon, la loba.

Ribs, etlinwill.

Rice, lice.

Rifle, calipeen.

Ring a, kwekwe.

Ripe, piah.

Ripe berries, piah olillie.

River, chuck.

Sack, le sak.

Saddle, la sell.

Sailor, ship-man.

Salmon berries, salmon olillie.

Salt, salt.

Sand, polallie.

Sandwich Islands, oihee.

Sash, la sanjel.

Saw, la gwin.

Say to, wah-wah.

Scissors, la seezo.

Sea, salt chuck.

Seal, olhyiu; Siwach cusho.

See to, nanitsh.

Sell to, mahkook.

Seven, sinomokst.

Sew to, mamook tupshin.

Shake to, toto; hullel.

Shame, shem.

Sharp, yahkisilt'h.

Sharpen to, mamook tsish.

She, her, yahka.

Sheep, la mooto.

Shell money (small size), koop-koop.

Shell money (large size), hykwa.

Shingle, lebahdo.

Shining, towagh.
Rooster, man la-pool.

Road, wayhut.

Roan-colored, sandelie.

Roast, mamook la pellah.

Rock, stone.

Rope, lope.

Rotton, poolie.

Round, lolo.

Rudder, boat opoots.

Rum, lum.

Run, coolie.

S.

Ship, ship.

Shirt, shirt.

Shoes, shoes.

Shoot to, mamook poh.

Short, yutesket.

Shot pouch, kalitan le sac; tsolepat.

Shot, shot; tenas le bal.

Shout to, hyas wahwah.

Shovel, la pell.

Shut to, ikpooie.

Sick, sick.

Sift to, toto.

Sight in, klah.

Silk, la sway.

Silver, t'kope chickamin.

Similar, kahkwa.

Since, kimta.

Sing to, shantie.

Sit to, mitlite.

Sister, kahpho, if older than

the speaker; ats, if

younger.

Six, taghum.

Skin, skin.

Skunk, hum opoots; piupiu.

Sky, koosagh.

Slave, eleitie; mist-shimus.

Sleep, moosum. 
Slowly, klahwa.

Small, tenas.

Smell, hum.

Smoke, smoke.

Snake, oluk.

Snow, snow, cole snass.

Soap, soap.

Soft, klimmin.

Sorrel colored, a sorrel horse, le blau.

Sorry, sick tum-tum.

Sour, kwates.

South, stegwaah.

Spade, la pell.

Speak to, wahwah.

Spill to, wagh.

Spirits, lum.

Split, tsugh.

Split to, tsugh.

Spectacles, dallah seah-host, or lakit seahhost.

Spit to, mamook tah.

Split, to become, chahko tsugh.

Spoon, spoon.

Spotted, tzum.

Spurs, le seeblo.

Squirrel, skwiskwis.

Stab to, klemahum.

Stand to, mitwit.
Stars, tsil-tsil.

Stay, mitlite.

Steal to, kapswalla.

Steam, smoke.

Steamer, piah ship.

Stick a, stick.

Stink, piupiu; humm.

Stirrup, sitlay.

Stockings, stocken, kushis.

Stone, stone.

Stop to, kopet.

Store, mahkook house.

Story, ehkahnam.

Straight, delate; sipah.

Strawberries, amotee.

Strong, Skookum.

Sturgeon, stuchin.

Stubborn, howklkult.

Sugar, le sook; shugah.

Summer, waum illahie.

Sun, sun; otelagh.

Sunday, Sunday.

Sunset, klip sun.

Suppose, spose.

Surprise, swah.

Swan, kahloke.

Sweep to, mamook bloom.

Sweet, tzee.

Swim, sitshum.

T.

Table, la tahb.

Tail, opoots.

Take to, iskum.

Take care, kloshe nanitsh.

Take off or out, mamook klah! mahsh.

Tale or story, yiem, ehkahnam.

Talk to, wahwah.

Tame, kwass.
Ten, tahtlum.

Tea, tea.

Testicle, stone.

Thank you, mahsy.

That, okook.

That way, yahwa, or kahkwa.

There, yahwa.

They, klaska.

Thick (as molasses) pitlilh. 
Thin (as a board), p'chih; pewhatie.

Thing, iktah.

Think, pittuck.

This, okook.

Thou, thy, thine, mika.

Thread, klapite; whalim.

Three, klone.

Throw away, mahsh.

Tide, chuck.

Tie to, kow.

Tight, kwult.

Tinware, malah.

Tip to, lagh.

Tired, till.

To, towards, kopa.

Tobacco, kinootl, kimoos.
Tomorrow, tomalla.

Tongue, lalong.

Tough, kull.

Trail, wayhut.

Trap, la piege.

Tree, stick.

Tree fallen, whim stick.

Trot to, tehteh.

Trout, tenas salmon.

Trousers, sakaleks.

True, delate.

Trunk, daessett.

Truth, delate wahwah.

Tub, tamolitsh.

Turnips, la-moo-ow.

Twine, tenas lope; kalpite.

To, twice, moxt.

\section{U.}

Uncle, tot.

Under, keekwillie.

Understand to, kumtuks.

Unhappy, sick tum-tum.

Untamed, le molo.

Untie to, mamook stoh; mahsh kow.

Up, saghalie.

Upset, kilapie.

Us, nesika.

V.

Venereal the, piah sick.

Venison, mowitsh.

Very, hyas.

Vessel, ship.

Vest, la west.

Vomit to, wagh.

W.

Wagon, tzig-tzig.

Wait, winapie.

Walk, cooley.

Wander to, tsolo.

Want to, tikke.

Warm, warm.

Wash to, mamook wash.

Watch a, tiktik.

Water, chuck.

Waterfall, tumwater.

We, nesika.

Week, ikt Sunday.

Weigh to, mamook till.

Wet, pahtl chuck.

Well then, abba; k'loshe kahkwa.

West, sun mitlite.

Whale, ehkolie; kwahnice.

What, iktah.

Wheat, sapolill.

Wheel, tzig-tzig.

When, kahta laylie.

Where, kah.

Whip, le whet. 
White, t'kope.

Who, klaksta.

Whole, lowullo.

Why, ikta mamook.

Wicked, mesatchie.

Wide, klakulh.

Wild, le molo.

Will the, tumtum.

Willow, eenastick.

Win to, tolo.

Wind, wind.

Window, glass.

Winter, cole illahie.

Wipe to, klakwun.

Wire, chickamin lope.
Wish to, tikke.

With, kopa.

Witchcraft, tamahnowus.

Without, halo.

Wolf, leloo.

Woman, klootchman.

Woman (old) lammieh.

Woman's gown, coat.

Wood, stick.

Work to, mamook.

Worn out, oleman.

Worthless, cultus.

Wound to, klemahun.

Write to, mamook tzum.

Writing, tzum.

\section{Y.}

Yard, ikt stick.

Year, ikt cole.

Yellow, kwakawak.

Yes, nawitka; ah-ah.

Yes indeed, nawitka.
Yesterday, tahlkie sun.

You (if plural), mesika.

You (singular), mika.

Your, yours, mesika.

Young, tenas.

\section{NUMERALS}

One, ikt.

Two, moxt.

Three, kloon.

Four, lah-kit.

Five, kwinnum.

Six, taghum.

Seven, sinnamoxt.

Eight, stotekin.
Nine, kwaist.

Ten, tahtlum.

Eleven, tahtlum pe ikt.

Twelve, tahtlum pe moxt.

Twenty, moxt tahtlum.

Fiftv. 1-winnum tahtlum.

Hundred, tukamonuk. 
No. 1.

\section{HAVE YOU BEEN TO JESUS FOR THE CLEANSING POWER?}

Spose mika tikke klap kloosh tumtum, Mika wash kopa Jesus pilpil ;

Alkie mika kloosh kopa kanaway sun, Mika wash kopa Jesus pilpil.

Chorus :

Mika wash, Mika wash, Mika wash kopa Jesus pilpil ;

Mamook halo masatchie kopa mika tumtum, Mika wash kopa Jesus pilpil.

Spose tikke cooley kopa kloosh wayhut, Mika wash kowa Jesus pilpil;

Yaka tikke mika kwansum coley delate, Mika wash kopa Jesus pilpil.

Chorus.

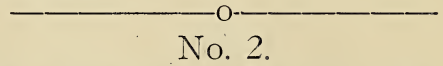

FOREVER HERE MY REST SHALL BE

\section{(Martyrdom.)}

Kwansum alke nika midlite, Kon'moxt mika Jesus; Pe nika kloosh tumtum, kewa, Yaka makook nika.

Nika Saviour, nika Tyee, Mika pilpil skookum; Mamook wash nika masatchie, Pe kwansum nika clean.

Wash nika pe alke kopet, Kanaway masatchie; Wake kopet nika lapee wash, Kloosh wash nika tumtum. 


\title{
No. 3.
}

\section{JESUS LOVER OF MY SOUL}

\author{
(Martyn.)
}

Saghalie Tyee tenass, Kloosh mika iskim nika;

Midlite yakwa hyas kull, Kakwa spose masatchie sun.

Kloosh mika ipsoot nika, Pe kopet masatchie sun; Mamook kloosh nika wayhut, Kopa mika illahee.

Halo waght nika tikke, Kopet ikt mika Jesus;

Halo mika mash nika, Kwansum potlatch nika kloosh.

Nawitka nika klap kloosh, Kopa mika, kloosh tyee; Kloosh ipsoot nika latet, Keekwillie mika lemah.

\section{No. 4. \\ ROCK OF AGES CLEFT FOR ME}

\section{(Ajalon.)}

Jesus nika kloosh tyee, Kloosh mika ipsoot nika; Ankutte mika pilpil, Mika mash kopa nika; Mamook kloosh nika tumtum, Pe wash nika okoak sun.

Spose nika cry kan'way sun, Spose nika skookum mamook ;

Halo alkie nika kloosh, Kopet mika kumtux save; Mika alkie save nika, halo ikta nika pay.

Kahta laylee nika wind, Kahta nika memaloose; Midlite kopa saghalie, Kah alke nanitch Mika; Jesus nika kloosh Tyee, Kloosh mamook ipsoot nika. 
No. 5.

\section{SUN OF MY SOUL, THOU SAVIOUR DEAR}

\section{(Hursley.)}

Jesus mika sun kopa nika,

Halo polakalie kah mika midlite;

Kloosh halo ikta masatchie,

Mamook ipsoot nika Tyee.

Spose nika midlite kopa sleep,

Kloosh nika halo kwass tumtum;

Pe mika kwansum kloosh nanitch,

Spose wake nika klap masatchie.

Kloonas ikta nika dzeepee,

Kloosh mika mash elip nika sleep;

Nika kwass tumtum memaloose,

Spose mika halo konamoxt.

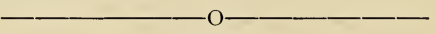

No. 6.

\section{O, HAPPY DAY THAT FIXED MY CHOICE}

O kloosh tumtum kopa ikt sum, Kah Jesus wash nika tumtum;

Kloosh nika mamook klahoney, Ikta Jesus halı tikke.

Kloosh tumtum, okoak sun, Kah Jesus wash nika tumtum;

Yaka mamook kumtux nika, Kahta spose alkie nika. pray;

Kloosh tumtum, okoak sun, Kah Jesus wash nika tumtum. 
No. 7.

\section{ABIDE WITH ME, FAST FALLS THE EVENTIDE.}

(Eventide.)

Jesus kloosh mika konamoxt nika, Wake laylee chako hyas polakilie; Spose halo klaxta kumtux help nika, Mika midlite skookum spose help nika.

Nika tikke mika kanaway sun, Kapit mika mamook delate tumtum; Halo klaxta kakwa tyee mika, Kloosh mika kwansum konamoxt nika.

Halo laylee nika midlite yakwa, Wake nika home midlite okoak illahee; Wake laylee alkie nika klap chee sun, Kah midlite Jesus yahwa nika home.

\section{No. 8.}

\section{COME THOU FOUNT OF EVERY BLESSING}

\section{(Nettleton.)}

Chako Jesus kopa nika, Mamook kumtux nika sing; Mika kwansum tikke nika, Mika delate nika king.

Chorus.

Nika hyas tikke Jesus, Nika tikke, nawitka; Nika tikke nika Saviour, Yaka waght tikke nika. 
Ankuttee nika masatchie,

Jesus yaka klap nika;

Yaka mash yaka kloosh pilpil,

Spose wake alkie lost nika.

Chorus.

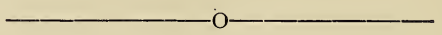

\section{No. 9.}

\section{AND CAN IT BE THAT I SHOULD GAIN?}

(Stella.)

Nawitka alkie nika klap, Kopa Jesus yaka pilpil?

Nawitka yaka memaloost, Spose mamook kilapie nika?

Kahta Mika hyas tumtum, Spose memaloose kopa nika.

Laylee nika tumtum midlite, Kakwa spose kow kopa lejob;

Mika mamook midwit nika, Kah laylee nika memaloose;

Mika mamook mash nika chain, Nika cooley kyimta Mika.

Wake kahta alta nika kwass, Jesus mamook delate nika,

Nika klap life kopa yaka, $\mathrm{Pe}$ alta nika kloosh tumtum ;

Delate alta nika cooley, Konamoxt Jesus nika Tyee. 
No. 10.

\section{HOW FIRM A FOUNDATION}

\section{(Portuguese.)}

Kahta nika skookum spose midlite tumtum, Kopa Saghalie Tyee, pe yaka tenass;

Ikta waght yaka wawa kopa nika?

Kopet kopa kahta Jesus memaloose.

Wake mika kwass Nika konamoxt mika,

Nika mika Tyee, Nika potlatch kloosh;

Nika mamook skookum kopa mika tumtum, Iskim Nika lemah, pe wake nika mash.

Spose kah mika cooley kopa hyas kull, Halo mika klap kopa sick tumtum;

Nika alkie konamoxt kah mika cooley,

Pe potlatch mika kloosh kopa konaway sun.

No. 11.

\section{WHOSOEVER WILL MAY COME}

Klaxta kumtux Jesus, hyas wawa; Mash okoak kloosh yiem kopa kanaway kah. Mamook kumtux kanaway klaxta tillicum, Klaxta tikke kloosh, chako.

Chorus.

Klaxta tikke kloosh, Klaxta tikke kloosh, Mash okoak kloosh yiem kopa kanaway kah ; Nesika kloosh Tyee halo tikke klaxta lost, Klaxta tikke kloosh chako. 
Klakta alkie chako wake mika kwass,

Alta yaka open okoak lapote;

Halo waght kloosh wayhut, kopet Jesus,

Klaxta tikke kloosh, chako.

Chorus.

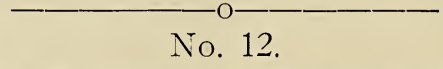

\section{THERE IS A FOUNTAIN FILLED WITH BLOOD}

(The Old Tune.)

Nawitka midlite kloosh pilpil, Ankuttee Jeșus mash, Pe klaxta midlite masatchie, Alkie kloosh spose yaka wash.

Kahta laylee nika nanitch, Jesus mika pilpil ;

Delate nika klap kloosh tumtum, Pe kopet nika till.

Alkie nika midlite saghalie, Pe sing kopa kloosh sing ;

Kahta Jesus makook nika, Kopa yaka pilpil.

$$
\text { No. } 13 .
$$

\section{DOWN IN THE VALLEY WITH MY SAVIOUR I WOULD GO}

Kah cooley Jesus alkie nika konamoxt, Kah midlite kloosh illahee, pe kloosh flowers midlite, Spose masatchie chako yaka kloosh nanitch nika, Pe kah yaka cooley alkie wake nika kwass.

Kyimta, Kyimta, nika kyimta Jesus, Kahta cooley kanaway kah nika konamoxt;

Kyimta, kyimta, nika kyimta Jesus Kahta yaka elip cooley nika kyimta. 
No. 14.

\section{ARE YOU WEARY? ARE YOU HEAVY HEARTED?}

Nawitka mika midlite till tumtum? Mamook kumtux Jesus;

Nawitka mika midlite sick tumtım? Kloosh mamook kumtux Jesus.

Chorus.

Mamook kumtux Jesus, Mamook kumtux Jesus,

Yaka delate tillicum;

Halo klaxta kakwa Jesus mika brother,

Kloosh mamook kumtux Jesus.

Kwansum chako chuck mika seahose? Mamook kumtux Jesus,

Midlite masatchie kopa mika tumtum? Kloosh mamook kumtux Jesus. Chorus.

Mika kwass spose alkie mika memaloose? Mamook kumtux Jesus,

Mika delate tikke kloosh tumtum? Kloosh mamook kumtux Jesus.

Chorus.

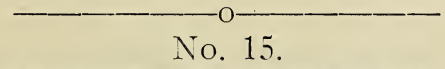

\section{HOW SWEET THE NAME OF JESUS SOUNDS}

\section{(Belmont.)}

Delate hyas dzee Jesus name, Kopa kloosh tillicum; Yaka mamook kloosh sick tumtum, Pe kopet kan'way kwass.

Hyas skookum Jesus kloosh name, Pe kloosh kopa nika : 
Kan'way kloosh ikta nika klap, Kopa Jesus kloosh name.

Jesus nika kloosh tillicum, Nika ow, nika king; Nika Tyee, nika wayhut, Iskim nika tumtum.

\section{No. 16.}

\section{TAKE THE NAME OF JESUS WITH YOU}

Klaxta midlite sick tumtum, Mika iskim Jesus name, Alkie mika chako skookum, Kanaway kah mika cooley.

\section{Chorus :}

Jesus name, hyas dzee, Mamook kloosh mika tumtum,

Jesus name, hyas dzee, Mamook kloosh mika tumtum.

Klaxta midlite hyas kull, Mika iskim Jesus name; Spose lejob chako skookum, Kloosh pray kopa Jesus name.

Chorus.

Ikta mamook kloosh nesika? Jesus hyas makook name;

Spose yaka iskim nesika, Kwansum kloosh nesik sing.

Chorus.

$$
\text { No. } 17 .
$$

\section{ALL PEOPLE THAT ON EARTH DO DWELL}

(Old 100.)

Klosh spose kanaway tillicum, Sing kopa Saghalee Tyee; Kwansum mamook kopa yaka:

Chako konamoxt kloosh tumtum. 
Kloosh kumtux Saghalee Tyee,

Wake klaxta yaka konamoxt;

Nesika yaka lamuto,

Kwansum kloosh nanitch nesika.

Delate kloosh nesika Tyee,

Pe kwansum long yaka tumtum;

Mamook tumtum kopa yaka:

Potlatch klosh wawa kanaway sun.

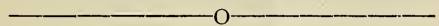

No. 18.

\section{CROWN HIM LORD OF ALL}

\section{(Miles Lane.)}

Hyas nesika kloosh tumtum, Kopa Jesus kloosh name; Nesika konamoxt angels, Spose mamook yaka king.

Klaxta ankuttee masatchee,

Pe Jesus mamook kloosh;

Lolo mesika kloosh tumtum,

Pe mamook yaka king.

Spose alkie nesika midlite, Konamoxt Saghalie Tillicum ; Alkie nesika kwansum sing, Pe mamook yaka king. 


\section{No. 19.}

\section{THERE IS A HAPPY LAND}

Midlite kloosh illahee, Siah, Siah;

Kah midlite kloosh tillicum, Siah, Siah:

Oh, kanaway klaska sing,

Jesus Christ Nesika King;

Hyas nesika sing, Kwansum, Kwansum.

Kloosh chako okoak, sun Chako, Chako;

Ikta kwansum moxt tumtum, Chako, Chako:

Alkie nesika kloosh,

Konamoxt mika Jesus,

Kah halo sick tumtum, Kwansum delate.

Midlite kloosh illahee, Kopa Saghalee;

Halo klaxta sick yahwa, Halo memaloose:

Oh, kwansum kloosh tumtum,

Kwansum, kwansum, kanaway sun;

Oh, kwansum kloosh tumtum, Kopa Saghalee.

$$
\text { No. } 20 .
$$

\section{NOTHING BUT THE BLOOD OF JESUS}

Ikta kumtux mash siah, Kanaway nesika masatchie? Ikta kumtux mamook delate, Kanaway nesika tumtum?

\section{Chorus :}

Kapit Jesus pilpil, Wash nika kakwa snow; Oh, hyas kloosh pilpil, Halo huloima ikta.

Ikta mamook Jesus mash, Ankuttee yaka pilpil? Yaka tikkee mamook delate, Kanaway nesika tumtum. Chorus. 
No. 21.

\section{COME YE SINNERS, POOR AND NEEDY}

(Guide.)

Chako mesika klahowya, Klaxta midlite masatchie; Jesus tikke save mesika, Pe potlatch mesika kloosh:

Yaka skookum, Yaka skookum, Yaka delate tikke save.

Chako mesika, hyak chako, Wake mesika moxt tumtum;

Saghalee Tyee tikke potlatch, Kopa kanaway kloosh ikta; Halo makook, Halo makook, Kultus potlatch mesika.

Klaxta midlite till tumtum, Kopa yaka masatchie; Spose mesika hyas laylee, Alkee chako kull tumtum: Hyak chako, Hyak chako, Alta Jesus tikke save.

\section{No. 22.}

\section{ARISE, MY SOUL, ARISE}

\section{(Lennox.)}

Midwit nika tumtum, Mash mika masatchie; Jesus yaka pilpil, Makook mika tumtum:

Yaka midwit kopa saghalie, Nika name tzum kopa yaka lemah. 
Jesus kwansum midlite, Saghalee illahee:

Yaka delate tikke, Kopa kanaway klaxta;

Nika kumtux Jesus pilpil, Wash kanaway klaxta tumtum.

Jesus kwansum yaka pray, Kopa yaka papa;

Spose mamook klahowya, Kopa nika tumtum:

Halo mika sollex Papa, Kwansum yaka hyas wawa.

\section{INDEX OF HYMNS}

Page

Abide With Me

Arise, My Soul, Arise............................................................ 47

Are You Weary, Are You Heavy Hearted?....................... 43

And Can It Be That I Should Gain?.................................... 40

All People That On Earth Do Dwell.................................. 44

Come Thou Fount of Every Blessing................................. 39

Crown Him Lord of All.......................................................... 45

Come, Ye Sinners, Poor and Needy.................................... 47

Down In the Valley With My Saviour I Would Go............ 42

Forever Here My Rest Shall Be............................................. 36

Have you been to Jesus for the Cleansing Power?............ $\quad 36$

How Firm a Foundation........................................................... 41

How Sweet the Name of Jesus Sounds................................... 43

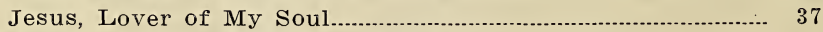

Nothing But the Blood of Jesus........................................ 46

O Happy Day that Fixed My Choice................................... 38

Rock of Ages, Cleft for Me................................................... 37

Sun of My Soul, Thou Saviour Dear................................... $\quad 38$

Take the Name of Jesus With You.................................. 44

There Is a Fountain Filled With Blood............................. 42

There Is a Happy Land..................................................... 46

Whosoever Will May Come..................................................... 41 
David Spencer Limited

Victoria Vancouver Nanaimo

Western Canada's

Mail Order

House

Write for Catalogue

David Spencer, Limited 
We Can Supply Your Every Need For

DIAMONDS, JEWELLERY, WATCHES, CLOCKS, SILVERWARE, BRASS, - AND LEATHER GOODS

Our Illustrated Catalogue, in which are pictured and described hundreds of articles from our fine stocks will be sent to your address F R F E upon request. Send your watches and jewellery for repairs. SATISFACTION GUARANTEED.

HENRY BIRKS \& SONS, LIMITED Jewellers and silversmiths

Geo. E. Trorey, Man. Dir. VANCOUVER, B.C.

KANAWAY TILLICUM TIKKE KLOOSH MUK-A-MUK

Ask YOUR Grocers for "MALKINS" BEST" Teas, Coffees; Canned and Dried Fruits; Canned Meats and Canned Salmon;

Spices; Fixtracts.

KANAWAY NESIKA IKTAS WAKE HYAS MAKOOK.

THE W. H. MALKIN CO.,

Wholesale Grocers, VANCOUVER, B. C. 